Journal of Bangladesh College of Physicians and Surgeons

Vol. 29, No. 2, April 2011

\title{
Pulmonary Hydatid Cysts and Tuberculosis in a Child - A Case Report
}

\author{
T BEGUM ${ }^{\mathrm{a}}$, S AFROZA ${ }^{\mathrm{b}}, \mathrm{F}$ AHMED $^{\mathrm{c}}$, AKM RAZZAQUE $^{\mathrm{d}}$, AA KIBRIA ${ }^{\mathrm{e}}$, A BAKI $^{\mathrm{f}}$, R ISLAM ${ }^{\mathrm{g}}$
}

\begin{abstract}
Summary:
A 7 years old male child presented with history of cough, fever, haemoptysis and chest pain for 2 years. On examination he was moderately pale and wasted. Respiratory system examination revealed features of consolidation in both lungs. His provisional diagnosis was pulmonary tuberculosis. TC was 1500/cumm ESR$70 \mathrm{~mm}$ in first hour, MT was negative and sputum for AFB was also negative. Radiological finding of chest revealed two large well defined dense opacities in both mid and lower zones of both lungs and there was no calcification or air fluid level. CT scan of chest showed large irregular enhancing mass lesion having air fluid
\end{abstract}

\section{Introduction:}

Hydatid disease (Echinococcus) is one of the most widespread, serious human cestode infection in the world $^{1,2}$.Its Public health problems occur in many areas, including counries of Central America and South America, Western and Southern/Southeastern Europe, the Middle East and North Africa, sub-Saharan countries, Russia and China ${ }^{2,3}$. Annual incidence rates of diagnosed human cases per100.000 inhabitants vary

a. Dr. Tamanna Begum. DCH, MD, Fellowship (Austrilia), FAAP. Associate Professor. Department of Paediatrics, Shahid Suhrawardy Medical College and Hospital, Dhaka.

b. Prof Syeda Afroza. FCPS, FRCP (Edin), DM Ed (UK), MM Ed (UK),Clinical fellow of Neonatiology (Edin). Professor and Head of the Dept of Paediatrics, Department of Paediatrics, Shahid Suhrawardy Medical College and Hospital, Dhaka.

c. Dr. Fahim Ahmed, MBBS, Medical Officer, Department of Paediatrics, Shahid Suhrawardy Medical College and Hospital, Dhaka.

d. Dr. AKM Razzaque. FCPS (Surgery) Associate Professor' NIDCH

e. Dr.Anwarul Anam Kibria. MS (Throcic Surgery), Rigistrar ,NICDH

f. Dr Abdul Baki. MBBS, FCPS-Part -1, Medical Officer

g. Rafiqual Islam, DCH, MPhil (International Health). Assistant Professor.

Address of Correspondence: Dr.Tamanna Begum, Associate Professor, Department of Paediatrics, Shahid Suhrawardy medical College and Hospital, Dhaka, E-mail-dr_tamanna@hotmail.com, dr_tbegum@yahoo.com.

Received: 21 June, 2009

Accecpted : 18 July, 2010 level in right lower zone, well defined cystic lesions in both lungs, no calcification was seen. On the basis of $x$ ray and $C T$ scan report we reviewed our diagnosis as pulmonary hydatid disease. Tablet albendazole was started preoperatively. The cyst was removed surgically and specimen was sent for histopathology. Report showed hydatid cyst and pulmonary tuberculosis. So confirmed diagnosis was Hydatid cyst and pulmonary tuberculosis.He was treated with antitubercular drugs and continuation of tablet Albendazole for 6 months. He was followed up regularly and was doing well.

(J Bangladesh Coll Phys Surg 2011; 29: 102-105)

widely, from less than 1 case per100,000 to high levels 2 , 3 . It is a zoonosis that is transmitted from domestic and wild members of canine family, who become infected after eating infected viscera and the host of the small adult worm. It is generally caused by the larval stage of dog tapeworm E.granulosus and man can become the intermediate host through contact with infected dogs or by ingesting contaminated food. ${ }^{5,6}$ Domestic animals such as sheep, goats, cattle and camels ingest Echinococcus granulosus eggs while grazing. Humans are accidental host and are usually infected with intermediate stage of the parasite by ingesting food or water contaminated with eggs or by direct contact with dogs $^{3,4}$ There are many studies of Hydatid disease in adult but only few articles regarding the rate and pattern of involvement in children have been published ${ }^{5,6}$. The liver and lungs are most frequently involved organs. Pulmonary disease appears to be more common in younger individuals but bilateral pulmonary involvement is relatively rare ${ }^{2,4,7}$ In a large series of 527 cases of hydatid disease from India, lungs were involved in $29 \%$ cases $^{5,6}$. In countries like India having high prevalence of pulmonary tuberculosis, an association of these two diseases, has not been frequently reported in medical litertaure ${ }^{8,9}$ 


\section{Case Report:}

A seven years old boy weighing $19 \mathrm{~kg}$, 3rd issue of nonconsanguinous parent hailing from Sirajgonj was admitted in the Paediatric ward of Shahid Suhrawardy Medical college and Hospital with the complaints of fever for 2 years, cough for 1 year, chest pain and difficulty in breathing for 2 months. According to statement of grandmother the child was reasonably well 2 years back then he developed fever which was intially irregular and low grade in nature but gradually became high grade and continuous for 4 months. Fever was relieved by taking anti-pyretic. He also developed cough for 1 year which was productive in nature, colour of the sputum was whitish, thin in consistency and was not foul smelling. There was history of hemoptysis for few occasions in last 2 months. He also developed difficulty in breathing.and chest pain. He had no history of contact with TB patient. He came from poor socioeconomic family, with poor housing and sanitation. Both parents were active and healthy. He was completely immunized according to EPI schedule. His milestone of development was age appropriate

On general examination, he was ill looking, moderately pale, not icteric, not cyanosed. Temperature was $102^{0}$ F and BCG mark was present. Lymphnodes were not enlarged. He was mildly stunted and moderately wasted according to WHO standard. His respiratory rate was 48/min, apex beat normal in position, breath sound vesicular and diminished in lower zone in both lungs and crepitation was present in mid and lower zone of both lung fields. Other systems revealed no abnormalities. Provisionally he was diagnosed as a case of pulmonary tuberculosis.

Investigations showed - Haemoglobin-10.8 gm/dl, ESR $70 \mathrm{~mm}$ in first hour, TC 11500/cumm, DC- N 72\%, L 18\%, M 02\%, E 10\% and PBF-microcytic hypocromic anemia. Radiological finding of chest showed - two large well defined dense opacities in both para cardiac regions in the lungs, there was no calcification or air and fluid levels fig 1).Ultrasonogram of the chest and hepatobiliary system showed multiple cystic area in chest cavities of both sides; the larger one is about $(7.5 X 5 \mathrm{~cm})$ detected in the left chest cavity, a hypoechoic area with irregular margin measuring (1.56X1.64) is seen in inferior aspect of right lobe of the liver (Fig-2). CT scan of the chest showed large irregular enhancing mass lesion having air fluid level in right lower zone, well defined cystic lesion in both lungs, no calcification was seen(fig -3), Total circulating Eosinophil count was 2400/cumm

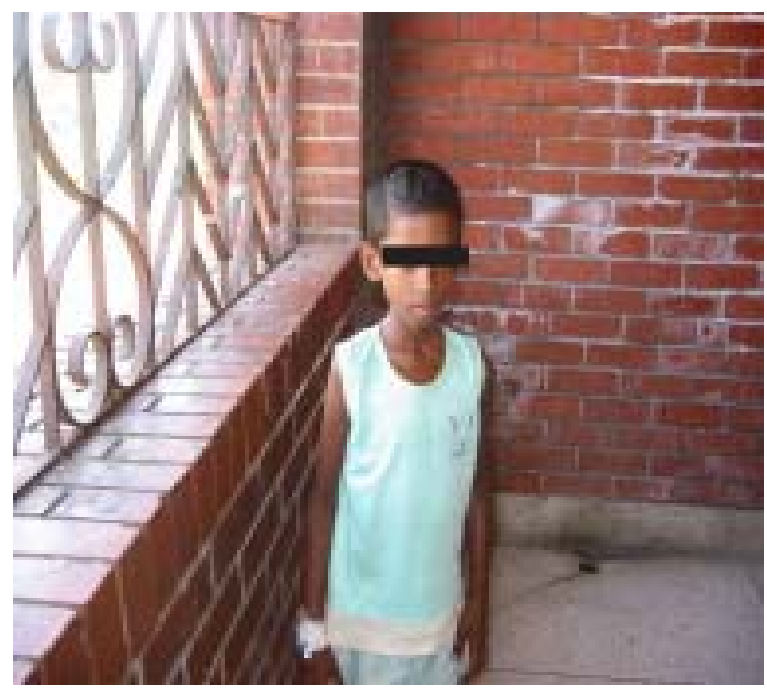

Fig.-1: Patient picture

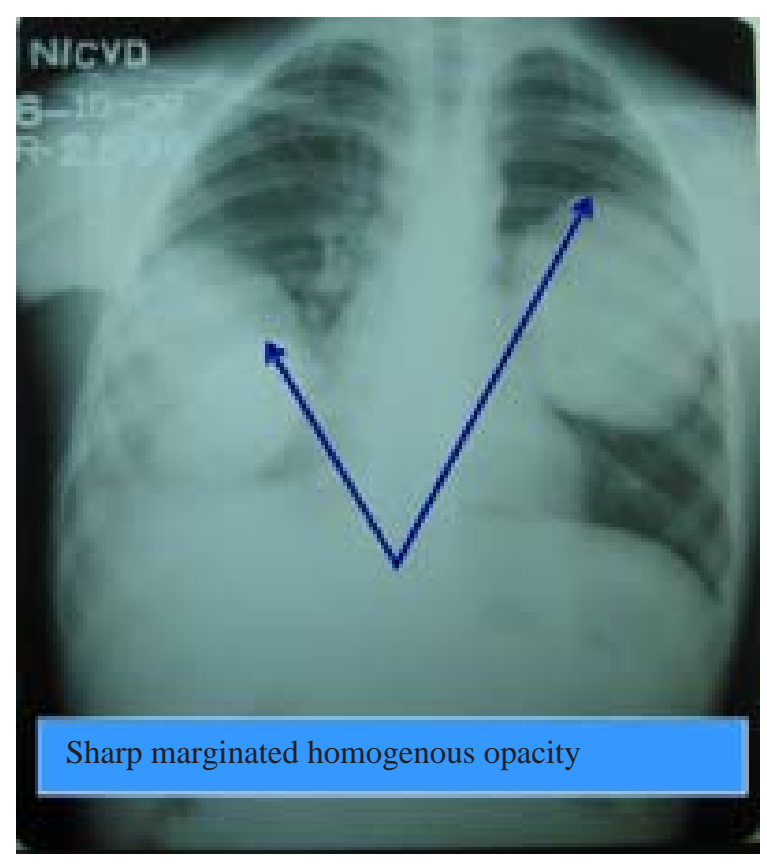

Fig.-2: $X$ - ray chest: Two large well defined dense opacities in both paracardiac regions. There was no calcification or air and fluild level. 
Beside nutritional support, broad spectrum antibiotics given, because of fever and neutrophilic leucocytosis. Albendazole $(15 \mathrm{mg} / \mathrm{kg})$ and hematinics were given as his PBF showed microcytic hypocromic anemia. His fever subsided but chest pain and difficult breathing persisted. He was refered to chest specialist and surgery was done in two settings (fig 4 first operation was excision of hydatid cyst through extended right posterior lateral thoracotomy followed by left upper lobectomy was done after one month. On histopathology-specimen of the lung tissue (fig-5), presents large area of caseating tubercles and section made from the cyst wall of laminated layer of hyalinized material.Diagnosis-

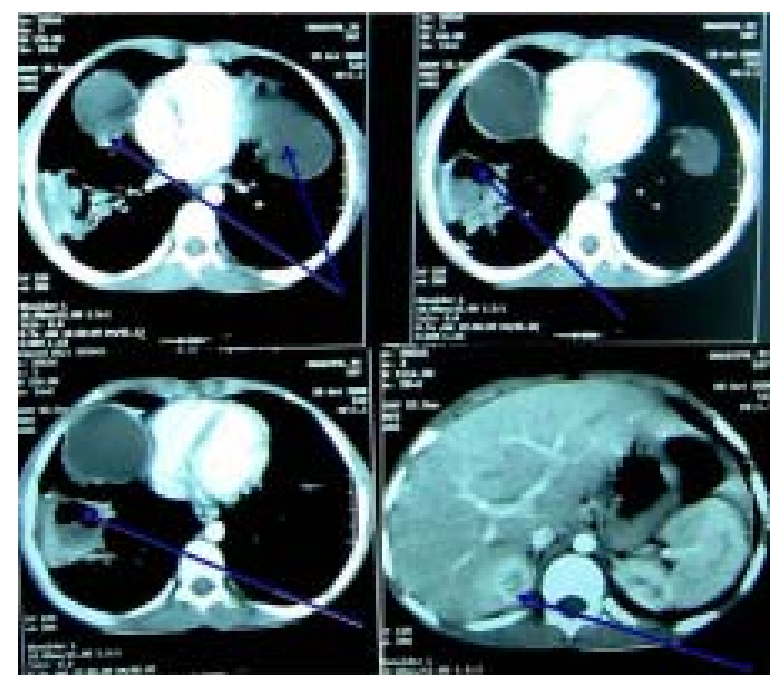

Fig.- 3: CT Scan of Chest: showed large irregular enhancing mass lesion having air fluid level in right lower zone, well defined cystic lesions in both lungs,no calcification was seen.

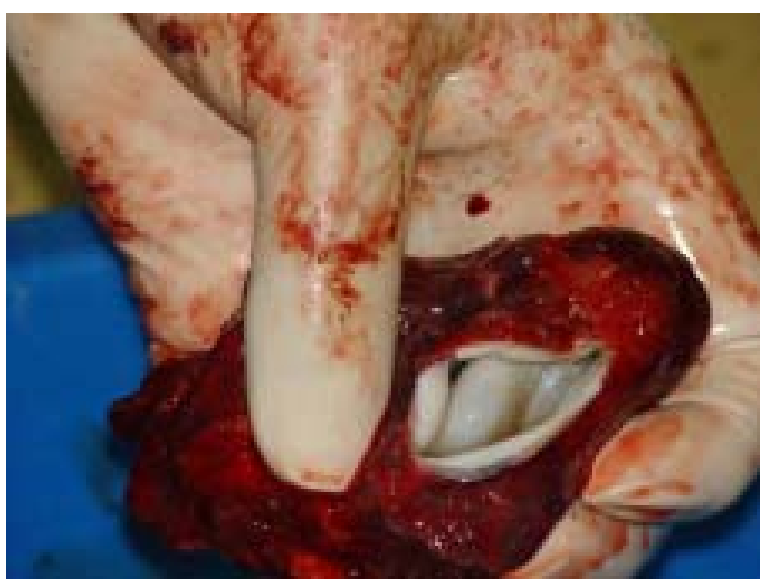

Fig.-4: Section of Lung showing hydatid cyst after operation ganulomatous inflammation, tuberculosis and hydatid cyst. He was treated with antitubercular drugs for 6 months with the doses of tab INH-10 mgkg, Rifampicin 10 mg/kg, Pyrazinamide 35 mg/kg daily(2HRZ+10 HR) along with albendazole $15 \mathrm{mg} / \mathrm{kg}$ daily for 6 months. He was clinically improved like weight gain, fever subsided and Hb increased after one month.

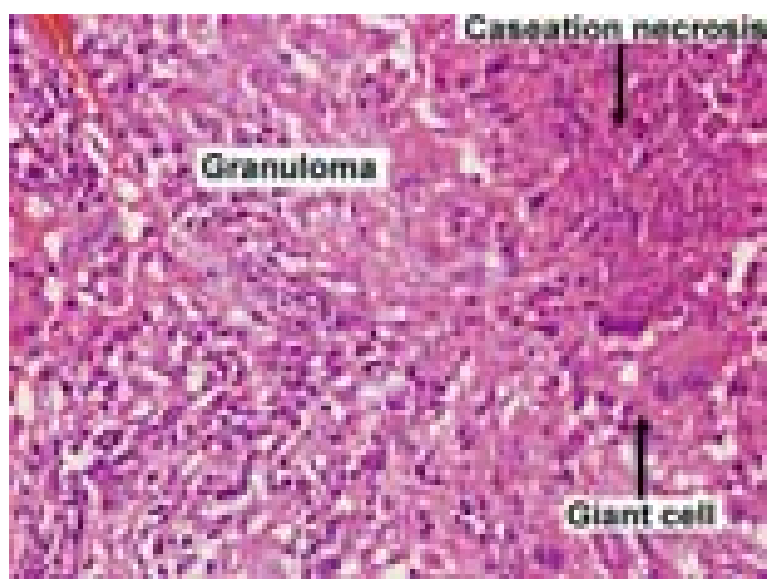

Fig.-5: Histopthology: of the lung specimen: granulomatous inflammation, area of caseating tubercles and section made from the cyst wall of laminated layer of hyalinized material. Diagnosis: Tuberculosis and hydatid cyst.

\section{Discussion}

Hydatid disease is an extensive epidemiological problem in developing countries like Iran, Egypt, and China ${ }^{3,4,5}$. The hydayid cyst in the liver is most common but can also be found in the lungs, kidney, spleen, nervous system and bone ${ }^{4,5}$. The frequency of hydatid disease was higher in male (58\%) than female (42\%) ${ }^{4,5}$. Signs and symptoms depend on cysts size and location which was shown in different studies as cough (92\%), fever(89\%), chest pain(42\%), breathlessnass(48\%) and haemoptysis ${ }^{4,5}$ Hydatid disease is a rare cause of hemoptysis and rare presentation of paediatric patient.The incidence of haemoptysis in children may be as high as $38 \% 5,6,7$, which is also present in our case. The most frequent affected organ in children is lung (77\%) whereas in adult liver is commonly affected organ 2,4, 5 . Similar finding was present in other studies The parasite is often acquired in childhood, but liver cysts require many years to become large enough to be detected or cause symptoms. Single organ involvement is present in $85-90 \%$ and more than $70 \%$ harbours a 
solitary cyst ${ }^{6.7,8}$. In children, the lungs appear to be the most common site as in the present case whereas $70 \%$ of the adults have disease in the right lobe of the liver ${ }^{5,6}$. It seems that in children scolices has more ability to pass from liver barriers than adults. It may be due to low density of the liver in children ${ }^{9,10}$. But combined lung and liver involvement is more frequent in children than adult $(18 \% \text { vs } 4 \%)^{8,9}$. Present case pulmonary hydatid disease affect both the lung like other studies the right lung in $60 \%$ cases, $30 \%$ multiple pulmonary cyst and about $4 \%$ bilateral cyst ${ }^{3,8}$. Although many patients are asymptomatic, some may occasionally expectorate the contents of cyst or develop sign and symptoms related to compression of surrounding structures. Most intact lung cysts are discovered incidentally on chest radiographs ${ }^{9,10}$. Studies showed well defined opacities in both paracardiac regions and there were no calcification or air fluid levels. CT scan of mediastinum revealed multiple large well defined intrapulmonary cystic masses in both lower lobes ${ }^{11,12}$. Similar finding was present in our case. Detection of antibody directed against specific Echinococcal antigen is useful in confirming a diagnosis but the false negative rate may be as high as $50 \%$ in cystic hydatid disease of the lungs ${ }^{4,6}$. ELISA for echinococcus was not done in our case because this is not specific and patient was poor. Few case reports were published hydatid disease with tuberculosis. Present case is also an example of this rare association. Some studies described hydatid disease as primarily a surgical disease ${ }^{13,14}$ Conservative surgical treatment like cystotomy, capitonage and other lung preserving surgical approaches like segmentectomy, lobectomy, wedge resection and enucleution were carried out in 65 of 72 children $(90 \%)^{10,11,12}$. However medical treatment may also be successful by benzimidazole drugs. Mebendazole and albendazole are the only antihelmenthic effective against cystic echinococcosis and probably treated before surgery ${ }^{3,8,10}$, which was followed in our case. More recently Keramidas et al treated 36 children with pulmonary echinococcus cysticus with oral mebendazole or albendazole, During this treatment 11 patients developed complications requiring surgery ${ }^{10,11}$. Surgical complications are less frequent in children and their outcome is better ${ }^{9,12}$. Recently few literatures highlight on percutaneous drainage of hydatid cyst popularly known as PAIR(Puncture Aspiration, Instilation of scolicidal agent and Reaspiration) has gained acceptance.This procedure is minimally invasive, cost effective, involves reduced hospital stay and less morbidity and mortality than surgery ${ }^{4,5}$.

\section{Conclusion:}

Hydatid disease of lungs is not so uncommon in Paediatric practice, but bilateral involvement is rare. Pulmonary tuberculosis is a co-existence with pulmonary hydatid cyst. Surgery is the mainstay of treatment for most patients with pulmonary hydatid disease in children but medical therapy for inoperative cyst with albendazole, mebendazole also suggestive.

\section{Refferences:}

1. Talaiezadeh AH, Maraght S. Hydatid diseases in children: A different pattern than adult Pak J Med Sci. 2006; 22:329332.

2. Brunetti E, Flica C.Echinococcosis Hydatid Cyst.eMedicineSpecialist infectious Diseases. http// www.yahoo.com. updateted May 28; 2008:1-12.

3. Ravinder K G, Rita G. Billateral Pulmonary Cyst in a Child. JK Science.2008; 10: 91-94.

4. Manish S, Saglheb A. PourakharB.Htdatid disease in Iranian children. J Microbiol Immnol Infet. 2007; 40: 428-431.

5. Kant S, Singh R, Bhatia R, Sanjay. Unusual presentation of Hydatid Disease. The Internet Journal of Pulmonary Medicine.2008;10:1-4

6. Brahim B, Mohamed M,Mouri, Abdeladf A, Ksaia, Amine A et al. Management of multiple echinococcus in childhood with albendazole and surgery. J Paediatric Surgery.2008, 43; 2024 -30 .

7. Kabiri EH, Kabiri M, Atoini F, Zidane A, Arslane A. Surgical treatment of pulmonary hydatid cysts in childhood. Arch Paediatric. 2006; 13: 1495 - 1499

8. Singh S, Vimesh P, Nadeem SA. Massive hemoptysis in children - unusual presentation in pulmonary hydatid disease.CTS Net. http//www.ctsnet.org; 2008:1-4.

9. Safet G, Zimida C, Lijaz P. Conservative surgical treatment of pulmonary hydatid disease in children. Medicinski artix 2007; 61: 1-3

10. Durakbasa CU, Sunder S, Schiratti V, Tireli GA, Tosyali AN, Murus M. Pulmonary hydatid disease in children: outcome of surgical treatment combinded with perioperative albendazole therapy. Paediatr Surg Int 2006, 22: 173 - 178.

11. Burhan K, Barkan V, Onem O, Bilcis and Demtrtas I. Conservative surgical treatment of pulmonary hydatid disease in Children: An Analysis of 35 Cases. Surgery Today 2002; 32: $779-783$

12. DhingraV.K, Rajpal S, KumarRaj. Concomitant presentation of pulmonary Tuberculosis and pulmonary Hydatid Disease in ATB Health worker.Indian J Allergy Immunol 2001: 15; 49-52.

13. J Seager, R.M.E Seal and PT Bray, Hydatid disease with hilar lymphadenopathy.Postgrad. Med.J. December 1978; 54: 809812.

14. Karande SC, Sheth SS, Lahiri KR, Shah MD.Co-existent hydatid disease and Pulmonary tuberculosis in a five years old girl. J Assoc P hysician India.1989;39: 353-4 